\title{
Unusual host carrying by a parasitoid wasp (Hymenoptera, Braconidae, Braconinae, Pycnobraconoides)
}

\author{
Donald L. J. Quicke', Steve Marshall² \\ I Division of Biology, Faculty of Life Sciences, Imperial College London, Silwood Park Campus, Ascot, Berkshi- \\ re SL5 7PY, UK and Department of Entomology, Natural History Museum, London SW7 5BD, UK 2 Insect \\ Systematics Lab, School of Environmental Sciences, 1216 Edmund C. Bovey Building, University of Guelph, \\ Guelph, ON, N1G 2W1, Canada
}

Corresponding author: Donald L. J. Quicke (d.quicke@imperial.ac.uk)

Academic editor: Stefan Schmidt| Received 27 September 2010 | Accepted 3 November 2010 | Published 8 February 2011

Citation: Quicke DLJ, Marshall S (2011) Unusual host carrying by a parasitoid wasp (Hymenoptera, Braconidae, Braconinae, Pycnobraconoides). Journal of Hymenoptera Research 20: 77-79. doi: 10.3897/jhr.29.868

Although many adult aculeate hymenopteran parasitoids physically manipulate their hosts, such as transferring them to more sheltered places prior to oviposition (Quicke 1997), such behaviour is highly unusual amongst non-aculeate parasitoid wasps. Thus, members of the superfamily Ichneumonoidea, along with most other parasitic, wasps, normally oviposit in their hosts in situ though one hyperparasitic species has been observed to physically haul on the silk thread of an escaping host larva to bring it within reach (Yeargan and Braman 1989).

We here report unusual carrying behaviour in the endemic Australian braconine wasp genus Pycnobraconoides. A single female wasp was observed and photographed at Barrington Tops National Park, Australia on 15th January 2010. Attention was drawn to it because it was carrying a case-making chrysomeline (Coleoptera, Chrysomelidae) larva, in its case, back and forth on a horizontal stem. The wasp was first observed for about ten minutes as it moved back and forth while using its front legs to hold the case beneath the stem approximately half a meter above the ground. A series of photographs shows the wasp periodically moving to a vertical stem before shifting the grip to the front and middle legs, and ovipositing (or at least probing with her ovipositor) in the case before shifting the case back to the front legs to carry it back and forth once again. 


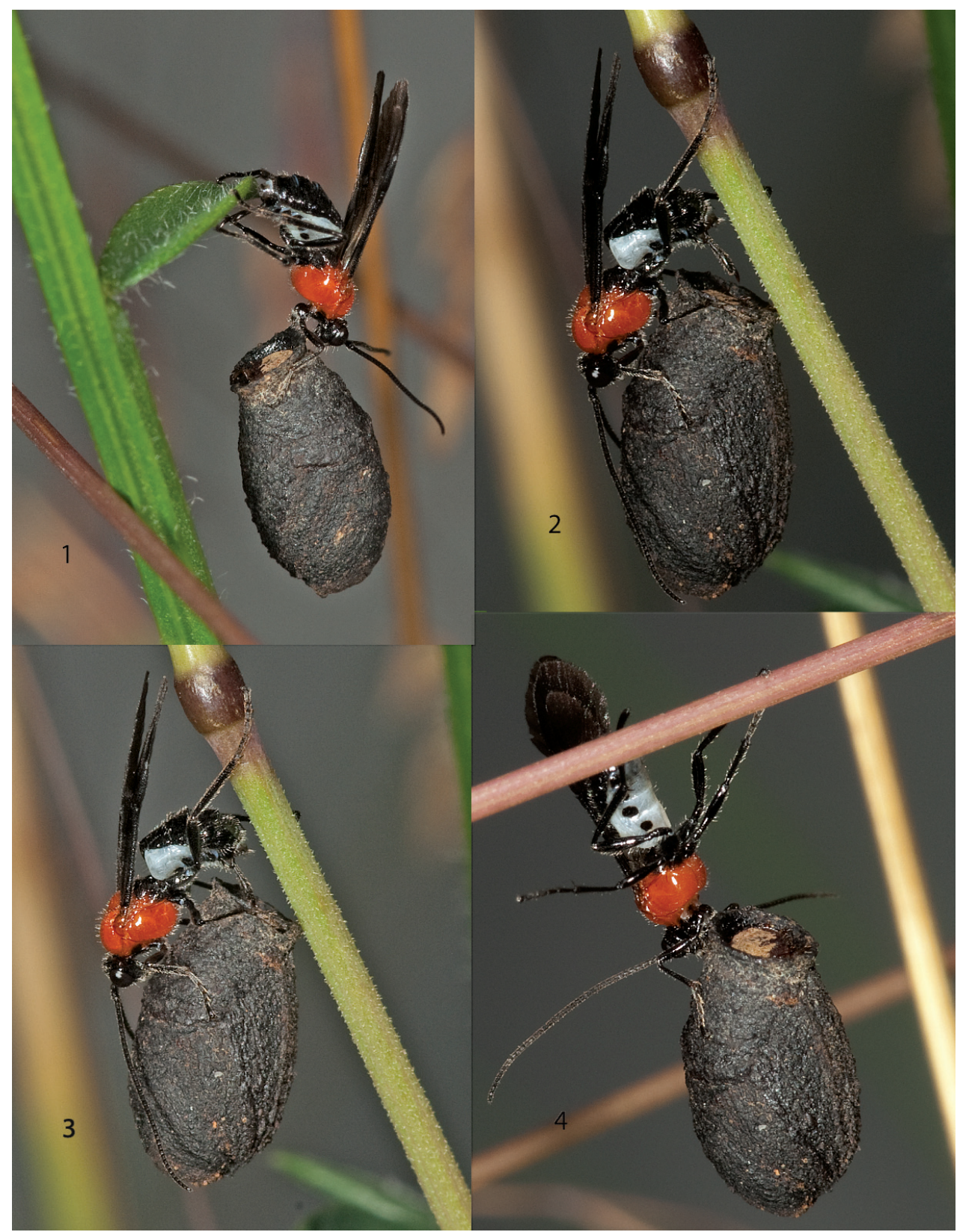

Figures I-4. Sequential photographs of female Pycnobraconoides sp. carrying and probing detached cryptocephaline chrysomelid beetle larva case.

After oviposition the case was again dangled below horizontal surfaces (leaves or stems) as the wasp gripped the leaf or stem with the hind and mid legs while suspending the relatively heavy host case with the front legs. The voucher specimen is now maintained in the University of Guelph. 
Pycnobraconoides has previously been reared from various cryptocephaline chrysomelid cases (Quicke and Ingram 1993). Its mode of oviposition was, however, unknown. Since members of this genus have a robust and pre-apically smoothly expanded ovipositor without conspicuous ventral valve serrations, which seems illadapted to penetrating hard cryptocephaline larval/pupal cases, it was likely that they had special, but unknown ways to access their host larvae for oviposition. One possible explanation for the observed case-carrying behaviour is that carrying induces the host chrysomelid larva to move its head and open up a channel for oviposition. In Notiopambolus Achterberg \& Quicke (1990), a similarly sized braconid genus that appears also to be a specialist parasitoid of cryptocephaline larval cases (Zaldivar-Riverón and Quicke 2002), the ovipositor is very different. In Notiopambolus it is very strongly dorso-ventrally compressed and upcurved, a condition that seems well suited to insertion between a 'clamped down' cryptocephaline case and the substrate, and which therefore probably enables the parasitoid to access the host larva in its case for envenomation and oviposition without recourse to physically moving it away.

\section{Acknowledgements}

We are grateful to Mike Sharkey for putting us into contact with one another.

\section{References}

Achterberg C van, Quicke DLJ (1990) A new genus of the tribe Pambolini from Australia (Hymenoptera, Braconidae). Zoologische Mededelingen, Leiden 64: 177-181.

Quicke DLJ (1997) Parasitic Wasps. Chapman \& Hall, London, 470 pp.

Quicke DLJ, Ingram SN (1993) Braconine wasps of Australia. Memoirs of the Queensland Museum 31: 299-336.

Yeargan KV, Braman SK (1989) Life history of the hyperparasitoid Mesochorus discitergus (Hymenoptera: Ichneumonidae) and tactics used to overcome the defensive behavior of the green cloverworm (Lepidoptera: Noctuidae). Annals of the Entomological Society of America 82: 393-398.

Zaldivar-Riverón A, Quicke DLJ (2002) First host record for the braconid wasp genus Notiopambolus Achterberg and Quicke (Pambolinae). Journal of Hymenoptera Research 11: 370-371. 